\title{
9 Rethinking objectification and its consequences
}

\author{
From substitution to sequence
}

\author{
Susanne Küchler
}

Anthropology has always struggled with accommodating objects into ethnographic analysis in ways that go beyond mirroring what people do and say. Despite its efforts to bring the object into the foreground of social analysis, and despite the fact that the material culture perspective has opened an invaluable new perspective on lived-in worlds richly exploited across the social and historical sciences, material culture has arguably not made a paradigm-shifting difference, theoretically and methodologically speaking. This is, I argue in this chapter, because the study of objects has remained firmly wedded to the social anthropological assumption of how objects work and what they do in society, an assumption framed within the theory of a dialectic constitution of subject and object relations. Productive of analyses that show how objects make people as much as people make objects, the definition of what has become known as the theory of objectification allows for an understanding of how social relations are articulated and maintained in the everyday. The work that made a lasting impact on how anthropology conventionally understands objectification and the difference it makes to society was Marcel Mauss's (2002[1925]) theory of the gift. Gifts, Mauss argued, are capable of substituting or standing in for persons, and are thus able to have an effect the reach of which is extended beyond the physical boundedness of a person. This definition of objectification, attentive to the classification of objects capable of standing in for persons became the foundational pillar of the anthropology of the twentieth century, framing the way it approaches objects to this day.

In fact, the definition of objectification as substitution set out by Mauss in his account of the socialising capabilities of the gift, has proved so productive in anthropological analyses that attempts at formulating an alternative definition have remained largely in the underground of the discipline. This chapter will retrace attempts at challenging the standard definition of objectification and set out the theoretical ideas that inform an alternative. This alternative is attentive to sequence and to relations between objects understood in temporal, rather than classificatory, terms. It argues that objects are not only capable of serving interpretation on account of their classificatory relation to one another, but to enable incantation on account of their capability to serve 


\section{Susanne Küchler}

as a model of sequences crucial to the understanding of the working of complex systems. The kind of complex operational systems that objects make tangible and visually accessible range from kinship to genealogy to resource and land use, all sharing a common trait of being part of distributive rather than extractive economies.

The search for an alternative definition of objectification met with resonance among anthropologists familiar with ecologies in which societies invest in prospective strategies and operatives that are predictable across time and space and that sustain political economies in which distribution reigns paramount. One such ecological niche is wider Oceania. To explain the relation between the idea of objectification, of a mathematical (recursive and predictable) nature of operational systems and the real world people inhabit, it is useful to recall the words of the geographer Tim Flannery (1994) whose Future Eaters set out the distinctiveness of the ideas that enabled the settlement of Australasia. Europeans, explains Flannery, came out of an environment conducive to the exercise and use of raw power, as the landscape was rich and extractable. By contrast, the flora, fauna, and human inhabitants of Australasia had to learn how to make a lot out of a little, to husband meagre resources by seeing how far they could be extended rather than how quickly they could be extracted. The distributive economies of wider Oceania thus have in common a concern with the mapping of sequences that underpin the complex operations of distribution, making life possible, and creating wealth by moving people and produce along rhizome-like networks (cf. Bird et al. 2019). Objects in this setting show off how predictable operations of distribution work and demonstrate the success of polities that claim ownership over resources.

The story of the alternative definition of objectification is one that involves the retelling of the history of theory in anthropology, following ethnographic research into wider Oceania. The notion that seemingly small societies create wealth and fame by extending themselves beyond the reach of persons via beautified objects such as canoes and decorative artefacts, is now commonplace in anthropology (Munn 1986; Bird et al. 2019). Ideas of (dividual) person and of (intellectual) property emerging from anthropology in Oceania (cf. Strathern 1988) quickly became now-classic counterpoints to an anthropology that had been framed by a very different set of ideas of personhood and property, emerging from the industrial context of mass production, where objects as substitutive of relations of labour (Forty 1986; Bourdieu 1984a). Alfred Gell's (1992) now classic paper - written for one of the early seminars on the anthropology of art held by a group of students in 1985 - profiled this double definition of objectification in his sharp critique of technology. Resonant of the work of Roy Wagner $(1975,1986)$ and the extended body of ethnographies emerging from Oceania, Gell's paper redirected theoretical thinking in anthropology to the question of what kind of work objects do and what this work does in society. In fact, the timing of the paper proved perfect as, with the end of Fordist mode production in the 1970s (Martin 
1992), the question of how objects capture the sequences and modalities of distribution, now at the heart of economies of production and consumption, quickly moved from a regional preoccupation of anthropologists to concern the mainstream in anthropology. Thirty years on, the questions now asked about infrastructures and operational sequences shown off by objects in a demonstrable fashion have been brought together in the present volume.

We might wonder why, in the face of ethnographies complicating the standard definition of objectification, the assumption that it can be reduced to substitution managed to prevail. One such explanation is given by Michel Foucault (1970) in his now classic, The Order of Things, in which he traces the theoretical and methodological appraisal of objects to Enlightenment's epistemological concern with Man (cf. Maniglier 2013). The study of objects as stand-ins for concepts and practices attended to by persons has drawn attention to the diversity of forms given to materials of the same category, in effect allowing concepts to be deduced from a comparison of forms within categories of objects, which in turn enable an inductive study of the technical processes of production. Branded by Tim Ingold (2012) as the 'hylomorphic model,' whose long tentacles reach down to Aristotle, the indexical qualities of form, allowing for an understanding of relations between persons and persons and things (Gell 1998: 148), have been commensurate with a rich body of method now synonymous with the study of material culture in anthropology (Tilley et al. 2006).

We arguably have had, however, hidden in plain sight, another still largely untold notion of objectification. Its tentacles reach to the depths of the anthropology of the Enlightenment, with the writings of Johann Gottfried Herder (1778) on emotion and cultural form and Gottfried Leibniz on the Theatre of Nature and Art (Bredekamp 2008), and forward into the twentieth century and the work of the art historians Aby Warburg and Walter Benjamin. This other sense of objectification is concerned. not with what is seen in the object and with forms that make visible relations of production (sehen), but with showing off in a demonstrable fashion (zeigen) what cannot be independently referenced from the object (cf. Ginzburg 2001). Objects of this kind officiate as diagrams, maps, or models that bring quantities into relation in a qualitative manner. Such objects capture, in their form, relations the nature of which entices what Gregory Bateson has famously called the method of 'double description' (1979: 79), in which abduction (the finding of similar forms) is followed by induction (comparison leading attention to shift from similarities to differences) and the formulation of higher-order concepts or normative rules (that explain differences). This means that relations immanent within such objects are not easily re-constructible, while they are intuitively recognisable and intersubjectively understandable.

The closest ethnographic account we have of such an alternative framing of objectification can be found in the little-known historical ethnography entitled Rockdale, which recounts the life of a rural Virginia town and its people on the eve of industrial revolution in America. Written by the 


\section{Susanne Küchler}

American anthropologist Anthony Wallace (1978), known for his work on religion, Rockdale recounts the practices and attentions to distribution, honed over centuries, that initially informed academic concern with the workings of machines.

Wallace describes in this study how the writing and receiving of letters was attended to with fervour in the days leading up to industrialisation. Letters, bound and kept, were tribute, trophy, or testimony to biographies whose strategic or fated unfolding they mapped. The life of letters attended to the sequences of sending and receiving letters, accompanied by an accoutrement of pens, tables, and chairs, of middlemen, and of collection points and modes of transportation. The operational capacity of machines initially was met with similar modalities of attention, directed as it were to the hidden sequences and connections, captured in diagrams, the improvement of which was the work of a new class of people known as the machinists. Wallace shows that machinists explained to each other the hidden workings of machines, not with words, but with drawings and models that rendered the inner workings visible and tactile (Wallace 1978: 237-238). The model or drawing of the machine at a size substantially smaller than the machine is shown to have been vital to allow the product of the machinists' thinking to be communicated in order to ensure that companions had approximately the same visual experience as the person operating the machine himself. What Wallace describes here is the importance of sharing the understanding of operational qualities among those working with and repairing machines, an understanding that drew on skills of mapping temporal relations via objects, in a not too dissimilar manner such as letters in pre-industrial times. Models and diagrams show off the workings of the machine, its invisible operational system, with an attention to the detail of connections that alone enabled subsequent breakthroughs in 'seeing' ways of improving its workings.

Wallace thus recovered for anthropology the way in which objectification can be seen to attend to operational thinking via the modelling of sequence, a notion that is also explored in Chapter 4 by Ludovic Coupaye. As models of operational sequence objects officiate as epistemic objects (see Hannah Knox and Rafael Schacter, Chapters 8 and 14). Unique to Wallace is that he draws our attention to the kind of epistemic work the modelling of operational thinking such objects accomplish. The machines he describes are demanding of care and attention - intellectual as well as physical - to stave off obsolescence (cleaning and repairing). They are also never completed, as every diagram or model is just a snapshot of a system that itself is opened to improvement as a result of the externalisation of its inner workings.

The objects that will be used in this chapter to explore the alternative definition of objectification are, thus, in more than one way, never singular and unique as they either capture different moments within an operational sequence or else project one of many possible perspectives onto the system as it is understood at present. This means that objects attuned to this alternative definition of objectification as sequence are demanding in that they require an 
ethnographic approach that is both time sensitive and time intensive. Time sensitive in that the attention to time, mapped in a modular fashion in an object, demands that we understand an object to unfurl as a sequence of images, the relations between which may show up as patterns of non-random variations when we attend to similarity and difference between one object and another.

By attending to the patterns of constancy and variation, we can then proceed inductively to arrive at an understanding of the normativity of sequence and proceed on to consider what kinds of operations the attention to sequence might capture. And this, more than anything, demands time - time to understand what operations in fact are being modelled. In Oceania, as we will see in the remaining part of this chapter, operations tended to differ widely and range from complex systems such as gardening, usufructury rights, and genealogy. What is attended to in any particular case study may also change over time and this, in turn, may lead to seemingly new ways of modelling sequences harnessing seemingly new materials and new technology. The alternative definition of objectification, therefore, demands sustained attention by the anthropologist to unpack clues, using objects and the images the systemic nature of which they reference obliquely, about operational systems that themselves demand to be attended to with equal dedication on the part of the anthropologist.

An example that illustrates the theoretical purchase, but also the methodological challenges, of a definition of objectification as modelling sequences underpinning operational systems is Yap stone money, described by the American explorer William Henry Furness III during his two months' visit to the Micronesian island in 1903 (Furness 1910; Martin 2013: 2-5). Given the brevity of his stay, Furness must have been gifted with extraordinarily powers of analytical imagination and clarity of observation as he was able to not just understand the complexity of the social system he found. He also understood how a system of credit and credit clearing that allowed a hierarchical system of relations to underpin the political economy of Yap society was modelled. The Yap had, he concluded, against his own expectations an economy based on money in the form of large, solid, and thick stone wheels, called 'fei,' ranging in size from between two feet to twelve feet. Although 'fei' were used to secure transactions, the stone wheels, taken from quarries by canoe from islands outside Yap territory, were never, or rarely, moved. This is because rather than serving as quasi commodities in barter-like exchange, they served to make manifest the potential for future transactions of the household, not its past executions. The measure of a stone wheel thus did not denote a relation to a hypothetical set of commodities that could be purchased with it. They were not mere stone coins, albeit of an unusual size, but instead were instrumental to a temporally structured system of credit and clearing - 'a tangible and visible record of outstanding credit the seller enjoyed with the rest of Yap' (Martin 2013: 12).

Furness concluded that the objects thus presented were not substitutes for other objects or forms of value, including persons in whose yard the boulders 
sat, but were manifestations of the system of social prestation itself. Yap stone money, differentiated in terms of scale, proportion, and multiplication, thus emerged as an objectification of distinct, temporally extended, forms of available credit. The idea that objects can serve to model sequences of exchange extended over a period of time and across a vast region was influential to my own analysis of objects that have been produced in their many thousands on an island known as New Ireland at the northernmost extension of the Bismarck Archipelago, which connects island Melanesia with Micronesia. Malanggan is a corpus of object, songs, and dances as well as sequences of rituals commencing with the burial of a person and concluding many years later in the carving, moulding, or weaving of a so-called malanggan effigy (Küchler 2002). Unlike the stone wheels carved by the Yap, malanggan effigies are not made to last, but are left to rot in the forest (woven effigies are burnt and moulded ones destroyed) or are sold to passing travellers via the island's mission stations that officiate as middlemen. The interest the fret-like carvings espoused among connoisseurs of Oceanic art led to collections numbering into the tens of thousands, the formal study of which had allowed me to formulate the hypothesis prior to fieldwork on the island. This hypothesis stated that effigies themselves were composite assemblages of motivic elements, and that the assemblage particular to each effigy was not random, but itself made reference to relations that were internal to the operational system of which they were a part. It took an extended period of ethnography study and renewed work on collections to understand that malanggan was not so much a system of credit bearing and credit clearing as a system the operation of which underpinned a complex leasehold system covering differentiated rights to land and its use. The transposition of a body after death into body politic, the image of which was intersubjectively shared by those participating in the 'work for the dead,' allowed a future directed and inherently resilient system of usufructury relations to span the entire island in a rhizomatic fashion that, like the effigies that serve to model it, was perpetually under construction. It took yet more perseverance to understand how it is that effigies reference such modular time maps, enabling people to navigate through time as confidently as they navigate the island, fending off unwanted trespass onto land as much as selectively forgetting retrospective leasehold relations to pave the way for prospective ones. Rather surprisingly, the act of binding turned out to be as critical as understanding the poly-perspectival nature of the knot and its manifold transformations (Küchler 2002), the geometric nature of which continued to inform hypotheses I began to formulate about seemingly very different objects fabricated elsewhere in Oceania.

The objects of Yap and island Melanesia are classic examples of objectification that frustrate attempts at conventional analysis, which assumes relations between persons and persons and things to be understandable via relations between objects, as these relations are referring back to themselves and to their systemic properties. Although the term is problematic in the connotations it conjures up, it is useful to recall the inflections given by the 
anthropologist Roy Wagner (1986), who refers to the self-referential nature and immanent relationality of objects as 'autistic' to emphasise the generative competencies inherent in such objects whose hold on imagination he was interested in understanding (1986: 11). I will expand on other examples in this chapter, each example showing, in the sense of showing off, the workings of a different complex system. The different kinds of understandings that their modelling makes possible will show that the analysis of objects that do the modelling is far from straightforward, demanding that we be sensitive to the workings of complex systems, the nature of which we may not be equipped to understand. Even if we are able to get our head around complex ecological systems, economic systems, genealogical systems, usufructuary systems, and kinships systems - to name just those that briefly will be touched upon in this chapter - the way their operational logic is attended to in the object might stretch the remit of our training and imagination to the breaking point.

While attention to sequence rather than to classification in our yet-tobe-fully appraised theory of objectification is fraught with difficulty, this chapter will try to explain the purchase this theory is set to deliver. In fact, by showing how objects and the particular qualities, symptomatic of their formal arrangement, attend to quantifiable data moved about in complex operational systems, we allow objects to be comparable to data compiled in charts and diagrams. A direct comparison can thus be made between the argument espoused in this chapter and the work of Antonia Walton on the aesthetics of 'good' science data. By studying objectification as qualitative modelling of complex systems, we can arguably begin to close the gap methodologically and theoretically between object collections and the understanding we bring to big data with the aim to understand better what kind of modelling works and the difference it makes to society. Perhaps most excitingly, we can begin to reach out to coding and computational modelling with a renewed zeal to match the thrill it offered as a new methodology back in the early 1960s, when Claude Levi-Strauss made the greatest inroads into our understanding of kinship systems and the transformational patterns of myth using early computing.

\section{The life of working models}

Rockdale is without a doubt not well known in anthropology. In fact, Rockdale is quite possibly the kind of books one will not ordinarily come across unless explicitly referred to it. Such is the power of personal reference; however, it should not surprise that the author's thinking had the most profound and lasting impact on the thinking of anthropologists working in places far removed from the small town in Virginia that was the subject of Wallace's archival research. This is because it so happened that two of the most prominent anthropologists working with acute theoretical intent on ethnographic data collected in Melanesia were Wallace's colleagues and friends. Of these, the Melanesianist Roy Wagner advanced Wallace's thinking in two of his most formative theoretical projects. These are Symbols that Stand 
for Themselves (1986), a book that followed his earlier work on The Invention of Culture (1975), while Frederick Damon tested Wallace's theory in his longterm research to understand the way people in island Melanesia model the constraints of living in a complex ecological system, recently published as Trees, Knots, and Outriggers: Environmental Knowledge in the Northeast Kula Ring (2016). The work of these two anthropologists has, in turn, had profound influence on a generation of anthropologists working in Melanesia.

In the introduction to Symbols that Stand for Themselves, Wagner considers the process of modelling in science and social science to show off familiar relations and orderings, in ways extended across analogous domains, as new understandings emerging through research that leads to a restructuring of the model. He uses the example of the idea of the double helix that is 'seen' in the sense of showing off and is thus able to inform the structure of the DNA, the subsequent remodelling of which instils a confidence in the model which, in turn, leads to 'paradigm certainty.' Wagner proposes that this modelling procedure, supporting paradigm certainty or trust, underpins the invention of culture (1986: 10-12). In paraphrasing the nature of modelling, he calls up the figurative usage of symbols, which cannot provide a literal field of reference, while 'figuring sympathetically by becoming itself that which it expresses' (ibid.: 6). Self-referencing in a generative manner - in short, the symbol that stands for itself - is simultaneously what it is and what it is about. As model, a sign is at once propositional and a resolution, bearing the imprint both of generic form and self-closure.

There are definite crossovers between Roy Wagner's thinking on modelling and the work of the anthropologist Patrice Maniglier (2006), whose ideas in The Enigmatic Life of the Sign are taking forward the immanent relationality and self-referencing of the sign in ways that constitute the ultimate paradigm shift in anthropological thinking. My own thinking on the peculiar role played by algebraic systems in supporting the generative and transformative qualities of objects (ethnographically informed by my work in the Pacific), has found a surprising echo in Maniglier's work, to which I will return in the concluding section of this chapter. For now, however, I turn to the work of Frederick Damon, which sets out the rather complex idea of self-referencing objects and their work as models of a system the complexity of which means that the model itself is perpetually under construction as much as is our understanding of it (cf. Ingold 2010).

Damon's book, Trees, Knots and Outriggers, on the Massim region of Papua New Guinea has been long in the making, as he himself admits (2016: 5). His interest in the canoe as model of a complex ecological system started when he first began to question how it is that people manage to live in the array of small and variously articulated islands clustered in the waters of Southwest Papua. To understand what people in fact know about the ecology of the area, and how these ideas are shared intersubjectively, Damon had to turn himself into a specialist of ethnobotany and ecology, with astrology to boot, as his research proceeded. In the small island environment of the Massim, a 
sailing vessel known as the kula canoe is central to connecting islands close enough to make a voyage possible and yet far enough to make travel by canoe arduous. The complex system of trade and exchange between the islands, each with its own ecological constraints on gardening and the harvesting of maritime resources, is the subject of the classical study of the kula (as the regional exchange system is known), carried out by Bronisław Malinowski (1922) in the early part of the twentieth century. Malinowski extensively reported on the minutia of the trade between the islands, constrained by both seasonal variations in growing patterns and the islands' peculiar environmental conditions that made some inhabitants entirely dependent upon trade of foodstuffs from other islands in exchange for pots and other articles produced by the resource-poor islands. There have been many subsequent studies of the kula canoe and of the relation between trade and the exchange of distinct valuables and their clockwise and anticlockwise exchange (cf. Leach and Leach 1983; Munn 1986).

Damon was the first to realise that the people there are attentive to the different types of trees from which the canoe is constructed and to the locations on an island where these trees grow. The selection of trees appropriate for particular parts of the canoe forms the backbone of the book, which is an exciting detective story into an unsuspected relationship of the trees, land, and canoes. The realisation that the structure of the canoe is mapped onto the island, and that this map reflects variations in seasonal and general intensity of gardening that significantly varies across islands, prompted further questions. Damon began to pursue the question of how a canoe is in fact working as a model of the spatio-temporal relations of seasonal resource use that enables islanders in the Massim to predict which island will have what kind of food ready for harvest at specific times of the year. The result is a fiendishly complicated study that shows canoes being refitted with new parts to serve as better models for subsequent sequences of its journey. The distribution of trees on the island follows the structure of a model canoe, invisible yet shared by all inhabitants as an idea that informs the relational nature of all actions in ways described by Nancy Munn (1986) as characteristic of the value-creating nature of the habitus.

Damon reported that he had almost given up ever being able to explain what he had understood about the intertwining of the operational qualities of the ecological system and the structure of the kula canoe when he read one of my own papers on the knot in Pacific imagination (Küchler 2003). What in fact had led to a breakthrough in my own understanding of how malanggan effigies model the usufructuary system was the decision to take seriously the fact that the effigy carvings did not look anything like the dead, whose passing they marked, even though photographs of people are available, and sculptors certainly were able to create life-like appearances from wood. What in fact was carved was a knot, or rather the inside of a knot transposed onto different dimensions to create systemic variations of one and the same object, casting shadows of its own life backwards and forward in time. In 
short, the knot and actions of binding turned out to be shaping the order of objects and their sequential relation to one another, allowing malanggan to model the operation of the leasing of land by relying on logic alone. It took me another few more years of immersing myself in the intricacies of differential geometry to understand the model I was dealing with even better. The object of malanggan, encompassing all possible past and future images, is seen as synonymous with the idea of membership in the leasehold system as each and every object is in fact analogous to the inside of an object such a Rubik's Cube that could be manipulated to bring in view multiple perspectives at the same time (Küchler 2014).

One of the complexities this approach to objects bring with it is that the analysis of working model requires a perspective unique to anthropology, namely the perspective encompassing the biographical life span. As anthropologists are rarely able to extend their research across time to allow for an appraisal of how time is mapped and modularised, understanding how one object unfurls into sequences of images that allow for retrospective and prospective strategies gets one partially there (cf. Gell 1993, 1998). Yet there is a further complexity, and this is that we need to take seriously the possibility that one object can be the gateway to understanding what in fact is manifold. This last section of the chapter takes this forward to explore a related challenge, namely, that objects made in Oceania have in common the peculiar capacity to 'show off' the capacity to contemplate a multiple as one, or as Anneliese Riles has said '[to] hold multiple levels of action in view at once' (Riles 1998: 379). The ideas exposed here are not for the faint hearted, touching as they do on some tricky geometry, thus raising the question of what the training of anthropologists should really encompass.

\section{Polymodality: geometry, transformation, and translation}

Sometimes the most perplexing aspect of anthropological theory is how well known a theoretical insight is and yet how little is done with it, for decades. In an era of postwar anthropology in Britain in which model building was all the rage, two publications stood out. The first was Edmund Leach's (1954) pathbreaking book, Political Systems of Highland Burma, in which he set out the use of operational, representational, and explanatory models underpinning the transformational nature of Kachin social structure. The second was LeviStrauss's (1969 [1962]) Elementary Structures of Kinship, which used computational analysis to unpack the logic informing complex kinship systems.

The influence of earlier texts on both these studies, which espoused the mathematical nature of working models of complex systems and the difference they make to society and culture is, however, not well known (Leach 1961). These texts are the posthumously published work (edited by none other than Levi-Strauss) of Abel Bernard Deacon (1934) on Malekula, an island of Vanuatu on the southern fringes of the Bismarck Archipelago mentioned earlier. Trained in Cambridge in natural sciences and studying for $\mathrm{PhD}$ in 
anthropology under Haddon, Deacon's findings led to a breakthrough in our understanding what the various objects attended to by Makelulans, from cats-cradle figures through to sand drawings and woven mats, were all about. Deacon's training in algebra and differential geometry enabled him to translate the myriad forms of patterns, woven from fibers and drawn into sand, spun into fibres and danced into the ground, into an algebraic system. Rather than being mere illustrations, pattern making in fact was shown up as a model the Malekulans think with, play with, and with which they seek to refine the operational sequences underpinning the extension of affinal relations.

For Levi-Strauss, Deacon's insights into the nature of the model and its relation to the operational qualities of the kinship system proved the decisive impetus for his later work on the Elementary Structures of Kinship (1969) that enabled anthropology to study kinship with a method and a rigor that was transformative to the discipline. For Deacon had in fact recognised the use in pattern making of an algebraic system of numbers and rules for their combination - the logic of which is known in mathematics as quaternion - that enables number series to be translated into geometric objects and back again. The peculiarity of the quaternion, the operation of which we know well from the Rubik's Cube, is that it enables one to envision changes to the spatial location of elements, in the formula and its geometric analogue, as reversible and as logically predictable. Sequences of 'moves' can be executed in the mind and the result be imaged from many perspectives at the same time, allowing the quaternion number system to be the code of choice for computing rotational objects digitally. The deployment of quaternion number systems proved revolutionary to Levi-Strauss who used it to unpack the transformational logic of myth and to understand the workings of complex kinship systems, the most complex of which he testified to exist in Malekula.

The legacy of these insights is acknowledged in the work of the anthropologist Knut Rio (2007) who has correctly established Malekulan sand drawings as an indigenous model and also highlighted the lack of a concern with classification and with the substitution of subjects in the making of objects. $\mathrm{He}$ does not, however, pick up on the mathematical and geometric ideas exposed by the kinship diagrams and the underpinning workings, in part because the drawings on which these ideas were based were by Deacon and not by the Malekulans. When we see that the drawings model the sequences of the system in much the same way as the objects upon which they are based model the idea of the operational system itself, we can see how fertile the conclusions to which Rio was drawn in fact are.

An example of an earlier study grappling with the type of logic underpinning an attention to objects that exposes their sequence in relation to one another is the work of Remo Guidieri and Francesco Pellizi (1981), who wrote a fabulous essay on the tree fern sculptures of Vanuatu. Their essay explores the relation between the figures and sequences of ritual that map out the stages men are required to go through to acquire ritual power. The figures do not serve to recall particular stages of the ritual, but rather they are there 


\section{Susanne Küchler}

to permit the contemplation of ideas underpinning a system that equates the highest rank with the position of the living dead. Their essay, seminal and poignant as it is, has been hugely significant to my own thinking about how objects model processes of transformation underpinning cosmologically charged genealogical systems across island societies in the Pacific, leading to my recent work on the coverlets stitched in the Cook Islands of Eastern Polynesia (Küchler and Eimke 2009; Küchler 2017).

The tivaivai coverlets whose stitched compositions of floral images and arborous patterns trap those looking at the coverlets as representations, as if they are made for seeing rather than contemplation. In fact, that the coverlets hold relations immanent within the assemblage of motivic elements and these relations affirm the cosmology of the body politic as much as they validate a concept of personhood that equates one with the many. Composed of iteratively replicated and transitively arranged self-similar motivic elements, themselves the product of recursive number systems and their non-commutative computation, tivaivai hold the clues to how they work and what they do locked within their own construction. Made as shrouds for the dead and fanned out during life in exchanges that punctuate the life cycle, tivaivai eventually are to be wrapped around the bodies of the dead in tomb-like graves, their assemblage quite literally reconstituting the relations cultivated by the deceased person in an artificial cloth body.

Invisible, and yet made for contemplation, the paradoxical nature of the tivaivai is paradigmatic to social life in the Cook Islands. Nothing matters more on these islands than a person's genealogical position in a complex relational matrix that connects foreign with homegrown ancestors to adjudicate who has rights to land and where, and who has rights to annunciate the resulting patterns of relations. The genealogical relational matrix that literally shapes a person's life is contemplated and shown off to others in the form of a pattern that is being stitched into the coverlets, itself remembered as a sequence of numbers made visual as coloured patches. While the sequences that reference the workings of the genealogical system are attended to as immanent within and inseparable from these patterns, the operational sequence of stitching itself varies across the life cycle of a household.

When a young family first sets out to make and gift coverlets, these tend to be of an applique type (ta-taura), meaning that a composite motivic element is arranged iteratively in a circular fashion on the planar surface of a monocoloured coverlet, showing off relations between households related to one another as affines, bound together by marriage. Relations that are not thought to have a lasting impact on the household are recognised with cut-out coverlets ( unfolds into an image with rotational symmetry in eight parts. It is only when her own children have left the household that a woman begins to stitch piecework coverlets (taorei) that recount the relations that connect the living with the ancestral generation. It is on a piecework coverlet that a young man must sit for his first ceremonial haircut while a genealogy is recited by his female 
relatives. It is the stitching of a piecework that a young girl learns who was adopted as baby into the household in ways that recount the arrival of the foreign ancestor. As the girl grows up she becomes the officiating head of a new household which, in genealogical terms, replicates all households that came before in a collapsing of time that is made visible and tangible in a demonstrative fashion in the piecework coverlet. Composed of iteratively replicated motivic elements, each in turn divided into a triangular shape, each part of a motivic element is stitched together by a different woman, following a precise sequence of coloured patches. The task is to stitch with the exact same tension as all the other women (in total 8 or 16 will be involved), working on one and the same coverlet so as to create a flat planar sheet that does not show unevenness. The 'oneness' of the tension and the exactitude of the replication of the pattern on the surface of the coverlet testify to a social body remade with every generation.

The principles of genealogy are attended in the Cook Islands as women stitch new coverlets to mark the extending of relations beyond the household to be eventually returned to the household. These sequences of unfolding and re-folding are also mirrored in the actions women perform upon the coverlets each time they are taken out of and returned to their storage trunks. We can also see the geometry of image-based polities at work when women create variations upon the pattern they have inherited from their adoptive ('feeding') mother in that the shape of a pattern is projected dimensionally, or in perspective, to show different views upon the same entity. A pattern is at once an algebraic entity and a geometric shape and, as such, immanent within the pattern, as inside and outside views are taken as an index of the subtle nuances of relations between persons and their households or simply to test the recipient's visual imagination and capacity to execute translations back to the geometry of the pattern. The rotational geometry of the pattern thus allows multiple views to be projected onto the surface of a coverlet, either successively or at the same time, almost as if the flat surface of the coverlet is itself a rotational three-dimensional object rather than the planar fabric it appears to be.

This aesthetic of the coverlet thus underscores the topological nature of its composition in that what is most important is not visible, but is reconstructed in the mind using imagination and the logic implicit in operations of genealogy. That it is the aesthetics of objects that is contemplated for its hidden reference to the workings of complex operations upon which life itself depends, is one of the often-missed conclusions of Alfred Gell's (1998) Art and Agency and inspired its writing. It is now time to return to the theme of this chapter, which concerned itself with recovering an alternative definition of objectification attending to sequence. The Cook Island coverlets also remind one of Wallace's writings about the machinists' attention to the complex operations of machines in their care. For it is the trust in the inner workings of a machine which was inseparably bound up with understanding and replicating the sequences of the operations critical to the work performed by each machine. 
Objects made to contemplate the work of the machine were shown by Wallace to make a difference, not just to the machinist's attention to the machine, enabling improvements to be made to its operational capacities over and above the care devoted to maintenance. Wallace shows how these objects (diagrams and models) also allow the community of machinists to separate as an identifiable social group from those who operated the machines. The difference that objects, attended to for the sequences they map and allow to contemplate, make to culture and society is central to the anthropological endeavour.

\section{Conclusion}

The examples this chapter has brought together are mere snapshots and sketches that outline how objects could attract attention, not because they stand in or substitute for persons, but because they attend to the inner workings of complex systems in a way that magically 'becomes what it expresses' (Wagner 1986: 6). We saw how this 'becoming' can involve the fracturing and sequential unfolding of parts of a composite image, capturing the transmission of partitioned land (in the case of the malanggan). And we saw the multi-perspectival shaping of a pattern stitched into coverlets turn many into one and one into many in ways that offer up an understanding of, and trust in, the faithful transmission of genealogy as the cosmological core of the image-based polities (in the case of the tivaivai). The distinction we conventionally make between diagrams, physical models, and objects was argued in this chapter to be unhelpful when engaging with the question of how objects can map out and enable the contemplation of sequences underpinning the operation of systems that may not be available to observation. The chapter argued that the consequences of a shift in attention away from classification of objects to the sequences of which they are a part, and which they themselves 'become' a part of as they make manifest this attention to sequence, may offer up a productive engagement with questions at the core of anthropological theory. Perhaps the greatest benefit, however, is that the definition of objectification allows for, and prompts, a literal retooling of methodology to aid the analysis of data that, like the operations they attend to, are inherently complex. It is in the hope to have inspired the rethinking of methods that this chapter has been written. 